\title{
Modeling of Unsteady Friction and Viscoelastic Damping in Piping Systems
}

\author{
C. Landry ${ }^{1}$, C. Nicolet ${ }^{2}$, A. Bergant ${ }^{3}$, A. Müller ${ }^{1}$, F. Avellan ${ }^{1}$ \\ ${ }^{1}$ Laboratory for hydraulic machines, EPFL, Avenue de Cour 33bis, 1007 Lausanne, \\ Switzerland \\ ${ }^{2}$ Power Vision engineering sàrl, Ch. des Champs-Courbes 1, 1024 Ecublens, \\ Switzerland \\ ${ }^{3}$ Litostroj Power d.o.o., Litostrojska 40, 1000 Ljubljana, Slovenia \\ E-mail: christian.landry@epfl.ch
}

\begin{abstract}
In real systems, the phenomena, such as pipe-wall viscoelasticity, unsteady friction or fluid structure interaction induce additional damping and dispersion of transient pressure waves than that defined by classical waterhammer. In this paper, unsteady friction models and viscoelastic damping models will be presented and a theoretical formulation of the viscoelastic damping in piping systems without cavitation will be developed. Firstly, the friction factor will be presented as the sum of the quasi-steady part and the unsteady part related to the instantaneous local acceleration and instantaneous convective acceleration. This unsteady friction model has been incorporated into the method of characteristic algorithm (MOC). Secondly, the damping will be defined in terms of viscoelastic effect attributed to a second viscosity $\mu$ '. This model is solved using the Finite Difference Method. Finally, numerical results from the unsteady friction and viscoelastic models are compared with results of laboratory measurements for waterhammer cases with low Reynolds number turbulent flows. This comparison validates the new viscoelastic model.
\end{abstract}

\section{Introduction}

The occurrence of pipe-wall viscoelasticity, unsteady friction or fluid structure interaction in fluidfilled pipes influences the evolving shapes of wave fronts as well as their damping. Indeed, these phenomena have a fundamental importance for fluid transient analysis and therefore the need of a more reliable analysis of pressure wave propagation as well as a better understanding of physical phenomena is the major motivation for the study of the hydraulic energy systems.

The transient flow occurs in pipelines when the pressure and flow rate change with time. Usually, pipe friction during transient events has been modeled using steady friction approximations, such as the Darcy-Weisbach friction equation. The quasi-steady state 1D model is characterized by a pseudouniform velocity distribution in each cross-section and a linear elastic behavior of the pipe material. This model is known for underestimating friction forces and overestimating pressure oscillations during the fast transient events [1]. In fact, the velocity profiles in unsteady-flow conditions show 
greater gradients, and thus greater shear stresses, than the corresponding values in steady-flow conditions [2].

To account for this lack of damping, unsteady friction models $J_{u}$ were introduced. The basic hyperbolic partial differential equations for one-dimensional unsteady pipe liquid flow $(|\vec{C}| \ll a)$ are, for an elementary pipe of length $d x$ and wave speed $a$ :

$$
\begin{array}{cc}
\frac{\partial H}{\partial t}+\frac{a^{2}}{A g} \frac{\partial Q}{\partial x}=0 & \text { Continuity equation } \\
\frac{\partial H}{\partial x}+\frac{1}{A g} \frac{\partial Q}{\partial t}+J_{s}+J_{u}=0 & \text { Momentum equation }
\end{array}
$$

Two types of models have been proposed in literature to describe fast transient events and to help the identification of dynamic effects: the unsteady friction 1D model and the viscoelastic model.

The unsteady friction 1D model is implemented by adding the unsteady-friction effect due to the non-uniformity of velocity profiles during fast transients. This model includes both the effects of local inertia and unsteady wall shear stress on flow, which several approximate formulas have been presented. Two distinct unsteady friction 1D models are considered in literature:

a) Instantaneous Acceleration Model

b) Convolution-Based Model

For the Instantaneous Acceleration Model, Brunone et al. [3] proposed the introduction of an additional term to evaluate unsteady friction losses with a decay coefficient $k$ related to the velocity distribution in the cross-section. This new model was theoretically justified by Axworthy et al. [4] on the basis of extended irreversible thermodynamics.

$$
J_{u}=\frac{k}{g A}\left(\frac{\partial Q}{\partial t}-a \frac{\partial Q}{\partial x}\right)
$$

where the coefficient $k$ is either experimentally calibrated or determined numerically from 2D model [5]. Brunone et al. [6] consider a $k$ coefficient that varies with time and space. The coefficient $k$ for these models was back-calculated from a more complex unsteady model and, therefore, more complex behavior can be replicated. Pezzinga [7] proposed a generalization of this model to provide dissipation without regarding the position of the valve generating the closure. Further, Vítkovský et al. [8] showed that the Brunone model is not able to correctly reproduce the oscillation damping observed for waterhammer following a rapid valve opening. Considering these limits, a new formulation of the unsteady friction is defined.

$$
J_{u}=\frac{k}{g A} \frac{\partial Q}{\partial t}+\operatorname{sign}(Q) \frac{k a}{g A}\left|\frac{\partial Q}{\partial x}\right|
$$

Finally, despite the generalizations of these kinds of models developed by different researchers, only the extreme values of the head oscillations can be reproduced, but not the shape. Moreover, all the dissipation effects not explicitly considered in the formulation of the model are included in the unsteady friction term. As a consequence, the calibrated values of $k$ vary somewhat, depending on the experimental conditions, and on the numerical implementation of the model.

The second 1D unsteady friction model considered is based on Zielke [9] with the convolution of past fluid accelerations and a weighting function. The weighting function is determined using the "frozen viscosity" assumption based on the steady-state viscosity distribution. Although realistic only for short 
time, this assumption is often acceptable in practice because the periods when unsteadiness has a strong influence on wall shear stresses can be very short-lived. Zielke [9] determined the weighting function for laminar flows. Unfortunately the computer solution of the convolution is time consuming. Trikha [10] simplified Zeilke's model using exponential relations for simulating frequency dependent friction and thus improved computational speed, but at the expense of solution accuracy.

Vardy and Brown [11] determined a set of weighting functions for the convolution-based approach for smooth-pipe turbulent flows. Recently, Ghidaoui and Mansour [12] efficiently implemented the Vardy-Brown weighting function in the method of characteristics; however, like the Trikha [10] implementation of Zielke's weighting function, accuracy was decreased as a trade-off for increased computational speed.

$$
J_{u}=\frac{2 v}{A D^{2}} \sum_{k}\left[\left(\int_{0}^{T} \frac{\partial Q}{\partial t}(t) W_{k}(T-t) d t\right) \cdot e^{-\left(\frac{4 n_{k} v}{D^{2}}\right) \Delta t}+\frac{m_{k} \dot{Q} D^{2}}{4 n_{k} v}\left(1-e^{-\left(\frac{4 n_{k} v}{D^{2}}\right) \Delta t}\right)\right]
$$

where $v=$ kinematic viscosity and $W=$ weighting function

The viscoelastic model assumes a viscoelastic behavior of plastic pipe-walls in the transient-flow equations. Comparisons between experimental and computational results using the Method of Characteristics (MOC) enhance some effects that can be identified as the main sources of surge damping. Thus, this concept expounded in detail by Habán [13] attributes the unsteady dissipation effects to a volume viscosity or second viscosity $\mu^{\prime}$. Therefore, this model has been implemented to represent unsteady damping effect in SIMSEN simulation program [14]. Moreover, the future perspective of plastic pipes has recently induced the development of more accurate hydraulic transient models taking into account the viscoelastic behavior of these materials [15], [16], [7].

The aim of this paper consists of theoretical formulation of the viscoelastic damping in piping systems without cavitation based on both experimental tests and computer modeling. Thus, the first part will define the most commonly used unsteady friction model and the viscoelastic model already existing in the SIMSEN software. Then, the second part will establish the new formulation of the viscoelastic model. Finally, numerical results from the unsteady friction and viscoelastic models are compared with results of laboratory measurements for waterhammer cases with low Reynolds number turbulent flows published by Bergant et al. [17].

\section{Unsteady and Viscoelastic Models}

The Vítkovský unsteady model described by the equation 5 combines local inertia and wall friction unsteadiness. This model is implemented using the Vardy's analytically deduced shear decay coefficient $C^{*}$ [18] to predict the Vítkovský's friction coefficient $k$ rather than use the traditional trial and error method for estimating $k$.

$$
k=\sqrt{C^{*}} / 2
$$

Where :

$$
\begin{aligned}
& \text { - For laminar Flow }: \quad C^{*}=0.00476 \\
& \text { - For turbulent Flow }: C^{*}=\frac{12.86}{\operatorname{Re}^{\log _{10}\left(15.29 / \mathrm{Re}^{0.0567}\right)}}
\end{aligned}
$$

This unsteady friction model has been incorporated into the method of characteristic algorithm (MOC). This popular method is a simple and numerically efficient way of solving the unsteady flow equations. In essence, the MOC combines the momentum and the continuity expressions to form a compatibility equation in discharge $Q$ and head $H$. Moreover, to satisfy the characteristic relations, the $\mathrm{x}$-t grid is chosen to ensure $\Delta x= \pm a \Delta t$. 
A first viscoelastic model is already implemented in the SIMSEN software. This software was developed by the EPFL for the transient and steady-state simulation of the electrical power system and hydraulic power system. The modeling of the hydraulic components with electrical equivalents provides a high level of abstraction and allows for a rigorous formalism. In this software, the equations (1) and (2) are solved using the Finite Difference Method with 1st order centered scheme discretization in space and a scheme of Lax for the discharge variable. This discretization leads to a system of ordinary differential equations that can be represented as a T-shaped equivalent scheme [14] as presented in Fig. 1.
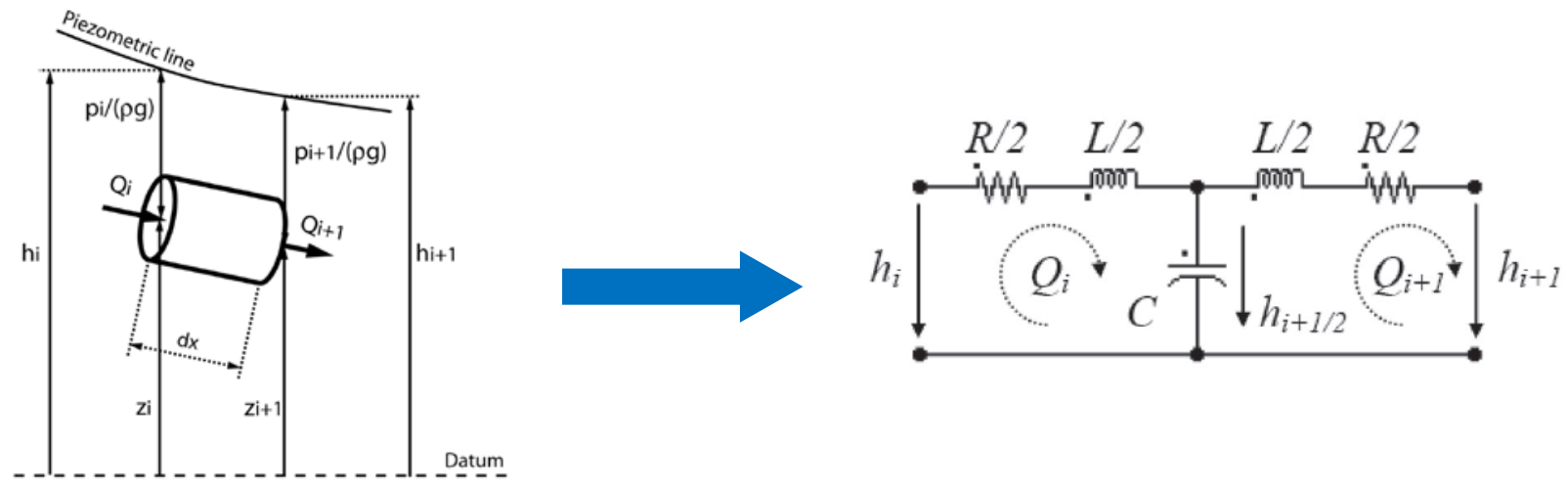

Figure 1. Representation of an elementary hydraulic pipe of length $d x$ and its equivalent circuit

The RLC parameters of the equivalent scheme are given by:

$$
R=\frac{\lambda|\bar{Q}| d x}{2 g D A^{2}}, \quad L=\frac{d x}{g A}, \quad C=\frac{g A d x}{a^{2}},
$$

where $\lambda$ is the local loss coefficient and $D$ is the diameter of the elementary pipe. The hydraulic resistance $R$, the hydraulic inductance $L$ and the hydraulic capacitance $C$ correspond respectively to energy losses, inertia and storage effects.

Moreover, in order to predict accurately pressure fluctuation amplitudes and system stability, it is necessary to take into account the viscoelastic behavior due to energy dissipation during the wall deflection. This additional dissipation is justified by the Navier-Stokes equations. Indeed, for a Newtonian isentropic fluid, the tangential viscous stresses $\tau_{r x}$ and normal viscous stresses $\tau_{x x}$ are defined in cylindrical coordinates by :

$$
\begin{gathered}
\tau_{r x}=\mu \frac{\partial C}{\partial r} \\
\tau_{x x}=\left(2 \mu+\mu^{\prime}\right) \frac{\partial C}{\partial x}
\end{gathered}
$$

where $\mu$ represents the viscosity and $\mu^{\prime}$ correspond to the expansion viscosity. Integration over a control volume $V$ of length $d x$ with a constant cross section $A$ is used to define the steady friction $J_{s}$ from the tangential viscous stresses and the diffusion term $J_{u}$ due to the compressibility of the fluid from normal viscous stresses.

$$
\begin{gathered}
J_{s}=\frac{\lambda Q|Q|}{2 g D A^{2}} \\
J_{u, v}=\frac{\mu^{\prime}}{A \rho D} \frac{\partial^{2} Q}{\partial x^{2}}
\end{gathered}
$$

This additional dissipation leads to a resistance in series with the capacitance. This viscoelastic resistance is accounting for both fluid and pipe material viscoelasticity and can be expressed as: 


$$
R_{v e}=\frac{\mu^{\prime}}{A \rho g d x}
$$

with $\mu^{\prime}$ the equivalent viscoelastic damping of both the fluid and the wall. Currently, this viscoelastic parameter is difficult to quantify and a comparison with experimental data are often essential to define its numerical value. This new viscoelastic parameter has a direct impact on the damping, but it does not take into account all the physics of the flow. The wave passage induces significant flow reversal near the wall. This flow reversal creates a large velocity gradient and thus a significant unsteady shear at the pipe-wall. It is widely recognized that the treatment of the wall friction as a static function of the mean velocity underestimates the wave attenuation at moderate and high frequencies. Inasmuch as the wall shear stress is not in phase with the mean velocity in pulsatile flow, the inertia term is modified by a factor $\zeta$ which is dependent on Reynolds number [1]. A first approximation of the $\zeta$ parameter is given by Wylie and Streeter [1]. However, a more accurate value can be obtained by analyzing the inertia term of the Vítkovský model. Indeed, this unsteady model has a good phase correlation with experimental data. Thus, it is possible to establish a new term of inductance for the viscoelastic model with the equation 6 :

$$
L_{v}=\frac{\zeta}{g A} \cong \frac{1+k}{g A}
$$

Finally, the new viscoelastic model implemented in SIMSEN software is described by adding a diffusion term $\mathrm{R}_{\mathrm{ve}}$ corresponding to the expansion viscosity and a new inductance $L_{v}$ corresponding to unsteady velocity profiles. To highlight the advantages and disadvantages of both models, numerical results from the unsteady friction and viscoelastic models are compared with results of laboratory measurements for waterhammer cases with low Reynolds number turbulent flows.

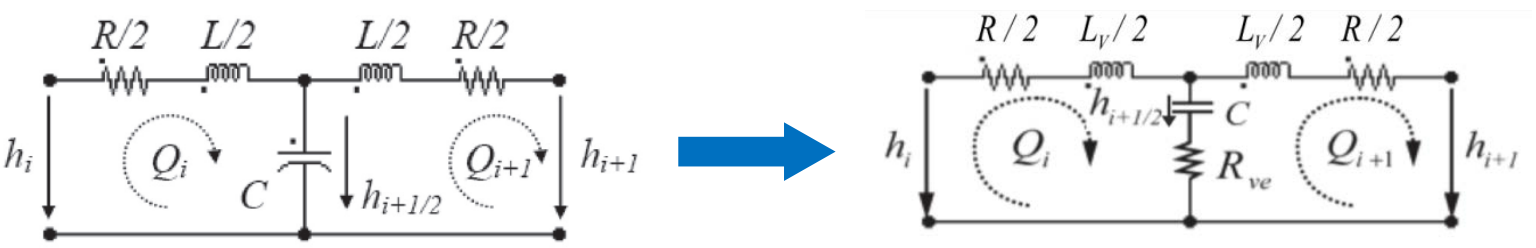

Figure 2. Representation of the new equivalent circuit for viscoelastic model

\section{Numerical and Experimental Results}

The apparatus presented in Figure 4 comprises a straight $37.23 \mathrm{~m}$ long sloping copper pipe of $22.1 \mathrm{~mm}$ internal diameter and $1.63 \mathrm{~mm}$ wall thickness connecting two pressurized tanks [17]. The static head in the upstream tank is equal to $32 \mathrm{~m}$. Waterhammer events in the apparatus are initiated by rapid closure of the ball valve. After 0.1 second, a fast closure of the valve is carried out by a torsional spring actuator providing a constant and repeatable valve closure time $t_{c}=0.009 \mathrm{~s}$. From an experimental point of view, three different steady state flow velocities $\left(C_{0}=0.1,0.2,0.3 \mathrm{~m} / \mathrm{s}\right)$ are analyzed and compared with numerical models to verify the performance of the unsteady friction and viscoelastic models. For each case, waterhammer wave speed $a=1319 \mathrm{~m} / \mathrm{s}$ was measured causing an oscillation frequency of $8.86 \mathrm{~Hz}$. Finally, computational and experimental results are compared at the valve, where pressure fluctuations are the highest.

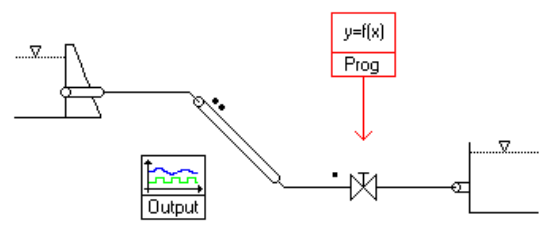

Figure 3. Representation of the SIMSEN model of the experimental device 


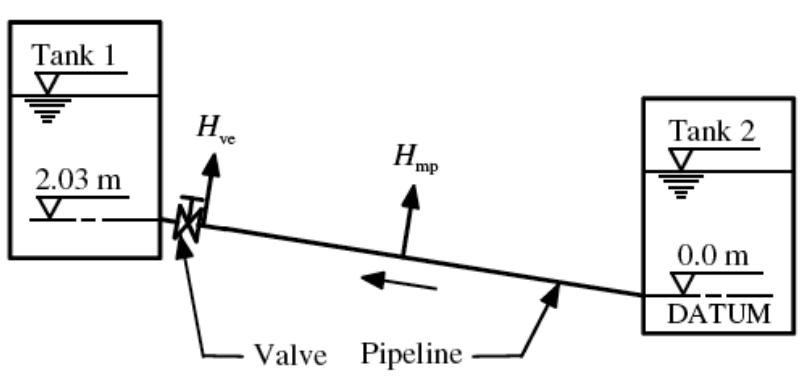

\begin{tabular}{|l|c|c|}
\hline Quantity & Value & Unit \\
\hline \hline Pipe length: & 37.23 & {$[\mathrm{~m}]$} \\
\hline Pipe diameter: & 0.0221 & {$[\mathrm{~m}]$} \\
\hline Thickness of wall pipe: & 0.0016 & {$[\mathrm{~m}]$} \\
\hline Pipe slope: & 3.2 & {$\left[^{\circ}\right]$} \\
\hline Head in Tank 2: & 22 & {$[\mathrm{~m}]$} \\
\hline Initial air void fraction: & $10^{-7}$ & {$[-]$} \\
\hline Valve closure time: & 0.009 & {$[\mathrm{~s}]$} \\
\hline Wave speed in liquid: & 1319 & {$[\mathrm{~m} / \mathrm{s}]$} \\
\hline
\end{tabular}

Figure 4. Test case experimental apparatus

For the first case, the experimental transient laminar flow velocity is equal to $0.1 \mathrm{~m} / \mathrm{s}$. The water temperature $\mathrm{T}_{\mathrm{w}}=15.4{ }^{\circ} \mathrm{C}$ imposes a Reynolds number $\mathrm{Re}=1870$. The Vítkovský model slightly underestimates the damping for the transient flow case. Indeed, after 1.4 second, the unsteady friction model overestimates by 3.4\% the experimental data. The match between the computed and measured results can be improved by using an empirically estimated $k$. Results from the viscoelastic model show an improvement in both attenuation and shape of the pressure head. The empirically estimated $\mu$ ' factor is equal to $8.26 \cdot 10^{5} \mathrm{~Pa} \cdot \mathrm{s}$.

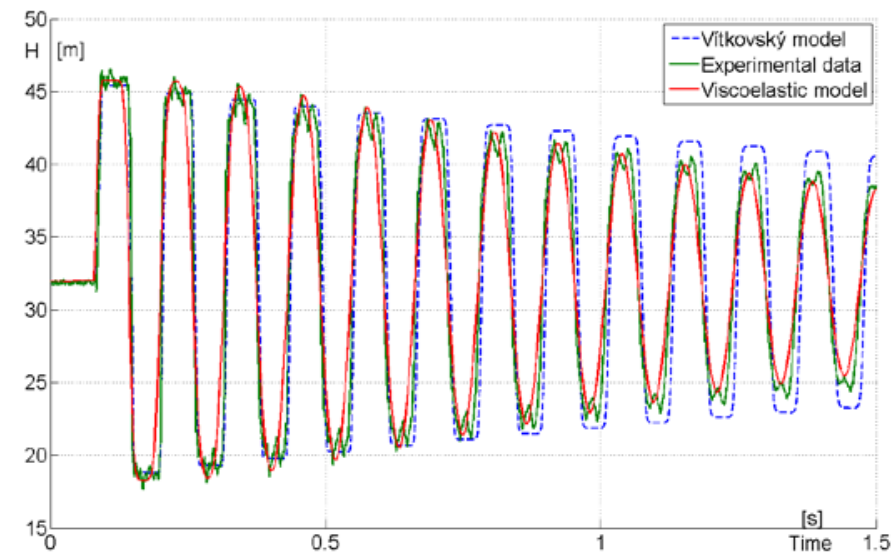

Figure 5. Comparison of piezometric head values at the valve between the numerical model and the experimental data for a steady velocity equal to $0.1 \mathrm{~m} / \mathrm{s}$

The two other experiments with initial flow velocities $C_{0}=0.2$ and $0.3 \mathrm{~m} / \mathrm{s}$ represent low Reynolds number turbulent flows. With a water temperature equal to $15.4{ }^{\circ} \mathrm{C}$, the corresponding Reynolds numbers are respectively 3750 and 5600 and the empirically estimated $\mu$ ' factor are respectively equal to $8.06 \cdot 10^{5} \mathrm{~Pa} \cdot \mathrm{s}$ and $7.76 \cdot 10^{5} \mathrm{~Pa} \cdot \mathrm{s}$. During the first oscillations, a slight overestimation of $4 \%$ of the head is computed by the viscoelastic model. This imperfection is subsequently corrected and the model describes a behavior in phase with a shape close to the experimental data. Conversely, the Vítkovský model describes well the first oscillations but underestimates the damping. The match between the computed and measured results can be improved by using an empirically estimated $k$. These effects are stronger when the flow velocity increases.

By observing the different values of the $\mu^{\prime}$ factor (see Table 1), it can be assumed that the value of this parameter is a function of the Reynolds number. 
Table 1. Summary of the main parameters of the viscoelastic model

\begin{tabular}{|l|c|c|c|}
\cline { 2 - 4 } \multicolumn{1}{c|}{} & \multicolumn{3}{c|}{ Reynolds Number [-] } \\
\cline { 2 - 4 } \multicolumn{1}{c|}{} & 1870 & 3750 & 5600 \\
\hline $\boldsymbol{k}[-]$ & 1.042 & 1.032 & 1.027 \\
\hline $\boldsymbol{\mu}[\mathbf{P a} \cdot \mathbf{s}]$ & $8.26 \cdot 10^{5}$ & $8.06 \cdot 10^{5}$ & $7.76 \cdot 10^{5}$ \\
\hline
\end{tabular}

However, as the three studied flows are in a restricted range values, it is difficult to extrapolate this assumption to higher Reynolds number. Moreover, according to Dörfler [19], unsteady friction may be represented by a bulk viscosity model which is inversely proportional with frequency. A comparison of the $\mu$ ' values presented in this paper with the regression proposed by Dörfler reinforces this concept of frequency dependence.

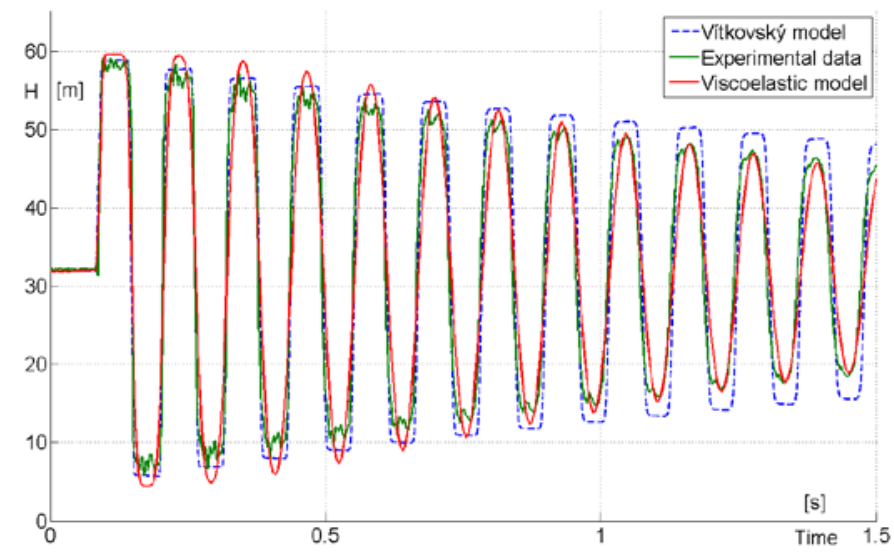

Figure 6. Comparison of piezometric head values at the valve between the numerical model and the experimental data for a steady velocity equal to $0.2 \mathrm{~m} / \mathrm{s}$

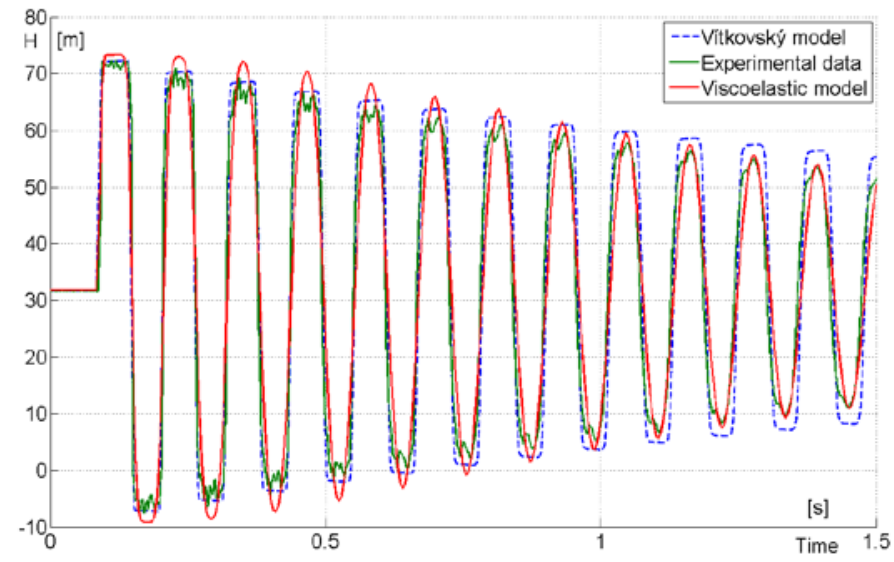

Figure 7. Comparison of piezometric head values at the valve between the numerical model and the experimental data for a steady velocity equal to $0.3 \mathrm{~m} / \mathrm{s}$

Unlike the viscoelastic model, the disadvantage of the unsteady friction model is its dependence on an experimental scale. Indeed, according to Duan et al. [20], the results show that the contribution of unsteady friction damping is important for small-scale (laboratories systems) but not for large-scale 
water supply and transport pipelines. Clearly, the relaxation of the velocity gradient diminishes the role of unsteady share. In fact, the faster is the relaxation time $\left(T_{d}=D^{2} / v_{k}\right)$, the less important is unsteady shear. Nevertheless, even when unsteady friction is not important for the wave envelope, it still imposes its signature on the wave shape.

Finally, we can conclude that two different physics can describe the same behavior. On the one hand, the unsteady friction may be represented by a friction term dependent on local inertia and wall friction unsteadiness. On the other hand, the unsteady friction may be represented by a dissipation term dependent on the expansion viscosity. However, according to Covas et al. [21], it is very difficult to make the distinction between the frictional and viscoelastic behavior.

\section{Conclusion}

Two types of models have been proposed in literature to describe fast transient events and to help the identification of dynamic effects: the unsteady state 1D model and the viscoelastic model. On the one hand, the viscoelastic model assumes a viscoelastic behavior of plastic pipe-walls in the transient-flow equations. A theoretical formulation of the viscoelastic damping in piping systems without cavitation was developed. The new viscoelastic model implemented in SIMSEN software is described by adding a diffusion term corresponding to the expansion viscosity and a new inductance corresponding to significant unsteady shear at the pipe-wall. The viscoelastic parameter $\mu$ ' is difficult to quantify and a comparison with experimental data are often essential to define its numerical value.

On the other hand, the unsteady phenomenon is also described by another physics combining local inertia and wall friction unsteadiness. Despite the generalizations of these kinds of models developed by different researchers, only the extreme values of the head oscillations can be reproduced, but not the shape. Moreover, all the dissipation effects not explicitly considered in the formulation of the model are included in the unsteady friction term. As a consequence, the calibrated values of $k$ vary somewhat, depending on the experimental conditions, and on the numerical implementation of the model. The results show that the contribution of unsteady friction damping is important for small-scale (laboratories systems) but not for large-scale water supply and transmission lines.

To highlight the advantages and disadvantages of both models, numerical results from the unsteady friction and viscoelastic models are compared with results of laboratory measurements for waterhammer cases with low Reynolds number turbulent flows. During the first oscillations, a slight overestimation of the head is computed by the new viscoelastic model, but this imperfection is subsequently corrected and the model describes a behavior in phase with a shape close to the experimental data. Conversely, the Vítkovský model describes well the first oscillations but underestimates the damping. The match between the computed and measured results can be improved by using an empirically estimated $k$.

Finally, we can conclude that two different physics can describe the same behavior. On the one hand, the unsteady friction may be represented by a friction term dependent on local inertia and wall friction unsteadiness. On the other hand, the unsteady friction may be represented by a dissipation term dependent on the expansion viscosity. However, it is very difficult to make the distinction between the frictional and viscoelastic behavior.

\section{Acknowledgments}

The authors would like to thank EOS Holding, Swisselectric Research and the Energy Program of The Ark, the Foundation for Innovation of Valais Canton, for their financial support. We would like to give special thanks to A. Bergant for the experimental data. 


\section{Nomenclature}

A Cross-section area $\left[\mathrm{m}^{2}\right]$

a Wave speed [m/s]

C Flow velocity $[\mathrm{m} / \mathrm{s}]$

C Hydraulic capacitance $\left[\mathrm{m}^{2}\right]$

$C^{*}$ Shear decay Coefficient [-]

$D$ Diameter [m]

E Specific hydraulic Energy [J/kg]

g Gravity [m/s $\left.\mathrm{s}^{2}\right]$

$H \quad$ Piezometric Head [m]

$J_{s} \quad$ Steady friction term [-]
$J_{u} \quad$ Unsteady friction term [-]

$k$ Decay coefficient [-]

$L \quad$ Hydraulic inductance $\left[\mathrm{s}^{2} / \mathrm{m}^{2}\right]$

$m_{k} \quad$ Coefficient of the $\mathrm{k}^{\text {th }}$ approximating term of $\mathrm{W}$

$n_{k} \quad$ Coefficient in exponent of the $\mathrm{k}^{\text {th }}$ approximating term of $\mathrm{W}$

$Q \quad$ Discharge $\left[\mathrm{m}^{3} / \mathrm{s}\right.$ ]

$R \quad$ Hydraulic resistance $\left[\mathrm{s} / \mathrm{m}^{2}\right]$
Re Reynolds number [-]

$R_{v e} \quad$ Viscoelastic resistance $\left[\mathrm{s} / \mathrm{m}^{2}\right]$

$W$ Weighting function

$\lambda \quad$ Local loss coefficient [-]

$\mu \quad$ Dynamic viscosity [Pa.s]

$\mu$, Expansion viscosity [Pa·s]

$v \quad$ Specific speed [-]

$v_{k} \quad$ Kinematic viscosity $\left[\mathrm{m}^{2} / \mathrm{s}\right]$

$\rho \quad$ Density $\left[\mathrm{kg} / \mathrm{m}^{3}\right]$

\section{References}

[1] Wylie, E.B. and Streeter, V.L. (1993), "Fluid Transients in Systems”, Prentice Hall, Englewood Cliffs, NJ.

[2] Vardy, A.E. and Hwang, K.L. (1991), “A Characteristic Model of Transient Friction in Pipes”, J. Hydraul. Res. Delft, The Netherlands 29(5), pp. 669-685.

[3] Brunone, B., Golia, U.M. and Greco, M. (1991), "Modeling of Fast Transients by Numerical Methods", Proceedings of the 9th International Conference on Hydraulic Transients with Water Column Separation, Valencia, Spain, pp. 273-281.

[4] Axworthy, D.H., Ghidaoui, M.S. and McInnis, D.A. (2000), "Extended Thermodynamics Derivation of Energy Dissipation in Unsteady Pipe Flow”, J. Hydraul. Eng. ASCE 126, pp. 276-287.

[5] Pezzinga, G. (2000), "Evaluation of Unsteady Flow Resistances by Quasi-2D or 1D Models”, J. Hydraul. Eng. ASCE 126, pp. 778-785.

[6] Brunone, B., Cacciamani, M., Calabresi, F., and Ferrante, M. (2003), “An investigation on unsteady-state friction in laminar flow", Pumps, electromechanical devices and systems applied to urban water management, Valencia, Spain.

[7] Pezzinga, G. (2002), "Unsteady Flow in Hydraulic Networks with Polymeric Additional Pipe”, J. Hydraul. Eng. ASCE 128(2), pp. 238-244.

[8] Vítkovský, J. P., Bergant, A., Simpson, A. R., and Vardy, A. E., Lambert, M. F. (2006), "Systematic evaluation of one-dimensional unsteady friction models in simple pipelines", J. Hydraul. Eng., 132(7), pp. 696-708.

[9] Zeilke, W. (1968), “Frequency-dependent Friction in Transient Pipe Flow”, J. Basic Eng. Trans. ASME Series D 90(1), pp. 109-115.

[10] Trikha, A.K. (1975), "An Efficient Method for Simulating Frequency-dependent Friction in Transient Liquid Flow”, J. Fluids Eng. Trans. ASME 97(1), pp. 97-105.

[11] Vardy, A.E. and Brown, J. (1995), “Transient, Turbulent, Smooth Pipe Friction”,J. Hydraul. Res. 33(4),pp. 435-456.

[12] Ghidaoui, M. S., and Mansour, S. (2002), "Efficient treatment of Vardy-Brown unsteady shear in pipe transients”, J. Hydraul. Eng., 128(1), pp. 102-112.

[13] Habán, V., Pochylý, F., Fialová, S., (2009), “The second viscosity of fluids”, Engineering Mechanics 2009, Prague, Czech Republic, Institute of Theoretical and Applied Mechanics, pp. 349-359.

[14] Nicolet, C. (2007), "Hydroacoustic modeling and numerical simulation of unsteady operation of hydroelectric system”, Ph. D. Thesis, EPFL (Ecole Polytechnique Fédérale de Lausanne), N³751, Lausanne (Switzerland)

[15] Covas, D., Stoianov, I., Maksimovi'c, C., Graham, N., Ramos, H. and Butler, D. (2002a), 
"Waterhammer in PE Pipes: Conceptual Model \& Exp. Analysis", Urban Water.

[16] Covas, D., Stoianov, I., Maksimovi'c, C., Graham, N., Ramos, H. and Butler, D. (2002b), "Hydraulic Transients in Polyethylene Pipes", EWRSA, ASCE, Virginia-EWRI, Roanoke.

[17] Bergant, A., Simpson, A., R., Vítkovský, J. P, (2001), "Developments in unsteady pipe flow friction modeling”, J. Hydraul. Res.,, Vol. 39

[18] Vardy, A.E. and Brown, J. (2002), "Transient Turbulent Friction in Smooth Pipe Flows", Journal of Sound and Vibration, Res. 259(5), pp. 1011-1036.

[19] Dörfler, P.K., (2011), "Pressure wave propagation and damping in a long penstock”, 4th International Meeting on Cavitation and Dynamic Problems in Hydraulic and Systems, Serbia

[20] Duan, H.-F., Ghidaoui, M.S., Lee, P. J., Tung, Y. K., "Relevance of Unsteady Friction to Pipe Size and Length in Pipe Fluid Transients”, J. Hydraul. Eng., February 2012.

[21] Covas, D., Stoianov, I., Mano, J.F., Ramos, H., Graham, N., Maksimovic, C., (2005), “The dynamic effect of pipe-wall viscoelasticity in hydraulic transients. Part II - Model development, calibration and verification”, J. Hydraul. Res.,, Vol. 43, pp.56-70 